\title{
Surface display as a functional screening platform for detecting enzymes active on PET
}

\author{
Sophia A. H. Heyde ${ }^{1,2}$, Jenny Arnling Bååth ${ }^{3}$, Peter Westh ${ }^{3}$, Morten H. H. Nørholm² and Kenneth Jensen ${ }^{1 *}$ (D)
}

\begin{abstract}
Poly(ethylene terephthalate) (PET) is the world's most abundant polyester plastic, and its ongoing accumulation in nature is causing a global environmental problem. Currently, the main recycling processes utilize thermomechanical or chemical means, resulting in the deterioration of the mechanical properties of PET. Consequently, polluting de novo synthesis remains preferred, creating the need for more efficient and bio-sustainable ways to hydrolyze the polymer. Recently, a PETase enzyme from the bacterium Ideonella sakaiensis was shown to facilitate PET biodegradation, albeit at slow rate. Engineering of more efficient PETases is required for industrial relevance, but progress is currently hampered by the dependency on intracellular expression in Escherichia coli. To create a more efficient screening platform in E. coli, we explore different surface display anchors for fast and easy assaying of PETase activity. We show that PETases can be functionally displayed on the bacterial cell surface, enabling screening of enzyme activity on PET microparticles - both while anchored to the cell and following solubilization of the enzymes.
\end{abstract}

Keywords: PETase, Ideonella sakaiensis, Surface display, Extracellular protein production, E. coli

\section{Introduction}

Poly(ethylene terephthalate) (PET) is one of the most commonly produced plastics (with an annual manufacturing of over 30 million tons), appreciated for its low cost, robustness, and high durability. This highly inert polyester consists of repeating units of the aromatic building block terephthalate (TPA) linked to ethylene glycol (EG) [1]. The inherent non-degradable properties that make PET an excellent material, unfortunately have the huge environmental drawback of substantial accumulation in landfills and in the ecosystem [2]. Increased build-up of plastic waste together with the fact that PET is produced from fossil feedstock call for efficient recycling and remediation strategies. These are currently dominated by thermo-mechanical and chemical means, with drawbacks like downcycling and the use of hazardous chemicals [3]. An environmentally friendly and less

\footnotetext{
*Correspondence: KHJN@novozymes.com

${ }^{1}$ Novozymes A/S, Biologiens Vej 2, 2800 Kgs. Lyngby, Denmark

Full list of author information is available at the end of the article
}

destructive recycling method is biodegradation, and recently, the interest in enzymatic PET degradation has increased tremendously [4].

The crystallinity and the limited accessibility to the ester linkages make PET unsusceptible to enzymatic hydrolysis, but a number of ester-cleaving enzymes have been reported active on the polyester [1]. The most promising candidates are classified as cutinases (EC 3.1.1.74), but the turnover rates are only low to moderate, reflecting that PET is an unnatural substrate for these enzymes [5]. A breakthrough in the field was the discovery of Ideonella sakaiensis in 2016, a bacterium able to utilize PET as its sole carbon source by converting it into TPA and EG, which was further metabolized. The initial catabolic pathway by I. sakaiensis involves two novel enzymes. The initial hydrolysis step of PET is facilitated by an extracellular enzyme homologous to cutinases, but with an extraordinary capability to degrade PET, hence classified as a PETase (EC 3.1.1.101). The hydrolysis products, of which Mono-(2-hydroxyethyl)terephthalic acid (MHET) comprises a major part, are transported into the periplasmic space, and degraded to TPA and EG 
by the second novel enzyme, classified as a MHETase (EC 3.1.1.102) [6].

However, even with the PETase's promising catalytic properties, problems with expression, purification and stability make it inadequate for industrial purposes $[7,8]$. Several engineering efforts have resulted in a few catalytically improved and more thermostable variants [7, 9-12]. Examples of improved variants of the I. sakaiensis PETase are the IsPETase ${ }^{\text {Austin }}$ [9], and Is PETase ${ }^{\text {Son }}$ [7]. The improvements in activity towards PET were achieved using two different strategies. In IsPETase ${ }^{\text {Austin }}$, the focus was to narrow the active site and to make it more cutinase-like by introducing the double mutation S238F and W159H, whereas the triple mutation S121E, D186H and $\mathrm{R} 280 \mathrm{~A}$ in Is PETase ${ }^{\mathrm{Son}}$ was centred around stabilizing loop regions, thereby improving the overall temperature stability. Irrespectively of the strategy, both variants were shown to clearly outcompete the wildtype PETase $\left(\right.$ Is PETase $\left.{ }^{\mathrm{WT}}\right)$.

Protein engineering efforts have all been done in $E$. coli, despite the complications imposed by the need for the release of intracellularly produced enzyme variants. Interestingly, only 2-3 mutations have been incorporated in the $I s$ PETase ${ }^{\text {Austin }}$, and $I s$ PETase ${ }^{\text {Son }}$ variants. One could speculate if use of a more amenable system, circumventing the limitations imposed by intracellular localization, would enable a more efficient protein engineering workflow for improving the IsPETase. As there is still no report of the PETase being successfully upscaled in an industrially relevant microbe, $E$. coli has remained the organism of choice, able to produce small amounts of the enzyme, albeit most of it ends up in inclusion bodies [8].

For large-scale production and screening of metagenomic samples for novel enzymes active on PET, there is a need for a robust and simple expression procedure. Bacterial surface display of enzymatically active proteins is a promising strategy to engineer whole-cell catalysts, but also to overcome the bottleneck of inefficient enzyme secretion and cytoplasmatic toxicity, enabling simplification of production procedures as well as downstream processes. Since the first attempts in the late ' 80 s , surface display is steadily gaining popularity and has been explored in most industrially relevant microbial platforms. Nonetheless, E. coli remains the most frequently used organism for surface display with a great variety of membrane anchors available [13]. Herein, we present a method for surface display expression of PETase variants using E. coli protein production strain BL21(DE3). This expression strategy allows for quick screening of the produced variants, where the lysis step and the formation of inclusion bodies are avoided. The procedure provides a convenient screening platform that could be applied to PETase variants in an industrially relevant organism suitable for integration into high throughput screening systems.

\section{Methods \\ Construction of expression vectors}

Genes encoding the three IsPETase variants (IsPETase ${ }^{\mathrm{WT}}$, Is PETase $\left.\mathrm{Austin}^{\text {, Is PETase }}{ }^{\mathrm{Son}}\right)[7,9,11]$ were synthesized according to the published codon-optimized sequence from Austin et al., 2018 [9] (final sequences can be found in Additional file 1: Sequence list) and cloned scarlessly into vectors $\mathrm{pKSD}$ :LppOmpA-NB and $\mathrm{pKSD}$ :NB-C-IgAP [14] using uracil-excision (USER) based cloning [15] and primers 1-7 (Additional file 1: Table S1) without their leader sequence and Met codon present. Sequences encoding TEV cleavage sites (ENLYFQ/G) were added in frame to either both sites of the enzyme sequence (IgAP module, STOP codon removed) or upstream of the N-terminus only (LppOmpA module, STOP codon intact) to enable cleavage from the surface anchor. We additionally added hexa-histidine tags $\mathrm{C}$-terminally prior to the terminal TEV cleavage site (C-IgAP module) or STOP codon (LppOmpA module) for purification of the different IsPETase variants after TEV cleavage. Both the TEV cleavage sites and hexa-histidine tags were fused directly to the coding sequence without use of linker sequences.

\section{Expression of surface-displayed IsPETase variants}

All pKSD expression vectors were transformed into BL21(DE3) competent E. coli (NEB) via heat shock and according to the manufacturer's description. Expression cultures were cultivated at $37^{\circ} \mathrm{C}$ in $500 \mathrm{ml}$ Erlenmeyer shake flasks without baffles in a culture volume of $50 \mathrm{~mL}$ lysogenic broth medium supplemented with $50 \mu \mathrm{g} / \mathrm{mL}$ Kanamycin. Cultures were induced at an optical density of $0.3-0.5$ at $600 \mathrm{~nm}$ via the addition of L-rhamnose (5 $\mathrm{mM}$ final concentration) and subsequently incubated for $20 \mathrm{~h}$ at $30{ }^{\circ} \mathrm{C}$ or $60 \mathrm{~h}$ at $16{ }^{\circ} \mathrm{C}$ (as stated) shaking at $250 \mathrm{rpm}$.

\section{GFP binding assay to assess surface display efficiency}

The nanobody:GFP binding assay was performed as previously described [14]. Surface display expression was terminated via centrifugation for $4 \mathrm{~min}$ at $2300 \mathrm{~g}$ and cells were resuspended in $50 \mathrm{mM}$ Tris buffer (pH 7.5). Purified GFP (for detailed purification protocol, see [14]) was added to a final concentration of $0.06 \mathrm{mg} / \mathrm{mL}$ and binding was allowed for $20 \mathrm{~min}$ at $30^{\circ} \mathrm{C}$ while shaking at 250 $300 \mathrm{rpm}$. Post GFP binding, cells were washed twice in 50 $\mathrm{mM}$ Tris buffer and $50 \mu \mathrm{L}$ of washed cell suspension was mixed with $100 \mu \mathrm{L} 50 \mathrm{mM}$ Tris buffer and transferred into a 96-well microtiter plate (Sigma-Aldrich). Optical density at $630 \mathrm{~nm}$ and GFP fluorescence (excitation: 485 
$\mathrm{nm}$, emission: $528 \mathrm{~nm}$ ) were measured in a SynergyH1 plate reader (BioTek) in transparent or opaque microtiter plates (Sigma-Aldrich), respectively.

\section{TEV cleavage}

For TEV cleavage of the surface-displayed IsPETase variants, cells were harvested for $5 \mathrm{~min}$ at $4000 \mathrm{~g}$, washed twice in $10 \mathrm{mM}$ Tris- $\mathrm{HCl}(\mathrm{pH} 7.5)$ and subsequently resuspended in the same buffer $(100 \mu \mathrm{L}$ per ODU). Commercial TEV protease (Sigma-Aldrich) was added to the cell suspension resulting in a final concentration of 1 Unit/ODU and the reaction incubated overnight at $4{ }^{\circ} \mathrm{C}$, rotating at $10 \mathrm{rpm}$ on a Blood Mixer Intelli-Mixer RM-2 S. Post incubation, cells were pelleted at $5000 \mathrm{~g}$ for $10 \mathrm{~min}$ and the supernatant was collected for activity analysis on para-nitrophenol $(p \mathrm{NP})$-acetate/butyrate and PET, and SDS-PAGE. Solubilized IsPETase variants were purified using His GraviTrap TALON (GE Healthcare Life Sciences) columns according to the manufacturer's instructions. In short, the supernatant was applied to a Ni-NTA agarose column. After washing with $20 \mathrm{mM}$ sodium phosphate buffer containing 500 $\mathrm{mM} \mathrm{NaCl}$ (buffer A) and $30 \mathrm{mM}$ imidazole, the bound proteins were eluted with $300 \mathrm{mM}$ imidazole in buffer A. Finally, the imidazole was removed using a PD-10 column (Amersham biosciences) equilibrated with $50 \mathrm{mM}$ HEPES (pH 8) buffer. The IsPETase ${ }^{\text {Austin }}$ purified control was purified as described in [16].

\section{p NP-acetate/butyrate activity assay}

$20 \mu \mathrm{L}$ whole cells $\left(\mathrm{OD}_{630}=0.1\right)$ displaying IsPETase variants on the cell surface, supernatant of washed cells after TEV cleavage, and purified IsPETase $(0.1 \mu \mathrm{g} / \mathrm{mL})$ were incubated with $1 \mathrm{mM} p \mathrm{NP}$-acetate or $p$ NP-butyrate (Sigma-Aldrich) in $50 \mathrm{mM}$ phosphate buffer ( $\mathrm{pH} 7)$ for $5 \mathrm{~min}$ at $24{ }^{\circ} \mathrm{C}$. The release of $p \mathrm{NP}$ was monitored at a wavelength of $405 \mathrm{~nm}$ in a SynergyH1 plate reader (BioTek). For $p \mathrm{NP}$-acetate assays on whole cells, E. coli BL21(DE3) cells not harbouring any of the surface-display expression vectors were used as negative control (NC). Since His-tag purified IsPETases were stored in 50 mM HEPES buffer ( $\mathrm{pH} 8$ ), the same buffer was respectively used as negative control for the $p \mathrm{NP}$-acetate assay on purified enzymes.

\section{Protein gels and western blot}

For in-gel fluorescence, cells were resuspended after nanobody:GFP binding to a concentration of 0.05 ODU/ $\mu \mathrm{L}$ and $20 \mu \mathrm{L}$ were mixed with $10 \mu \mathrm{L} 2 \times$ Laemmli sample buffer and $0.5 \mu \mathrm{L}$ benzonase nuclease ( $\geq 250$ units/ $\mu \mathrm{L}$, Sigma). $15 \mu \mathrm{L}$ of the whole sample were loaded onto a 4-20\% Criterion $^{\mathrm{TM}} \mathrm{TGX}^{\mathrm{TM}}$ gel (Bio-Rad) at non-denaturing conditions and run for $30 \mathrm{~min}$ at $200 \mathrm{~V}$. Fluorescent protein bands were visualized using UV light and a GelDoc XR + imaging system (Bio-rad). Total protein was assessed afterwards by staining with Novex SimplyBlue SafeStain. For IsPETase detection by western blot, samples were mixed with Laemmli sample buffer as described above, boiled for $5 \mathrm{~min}$ and separated via SDSPAGE (4-20\% Criterion ${ }^{\mathrm{TM}}$ TGX $^{\mathrm{TM}}$ gel (Bio-Rad) at denaturing conditions). Total protein was transferred onto a nitro cellulose membrane using the BBlot $^{\mathrm{TM}} 1$ gel transfer system (Invitrogen). Blots were blocked with TBS-1\% dried skim milk powder and developed with sequential incubations of mouse anti penta histidine tag ( $\alpha$-His) antibody (Bio-Rad, MCA5995P) and horse radish peroxidase (HRP) conjugated secondary goat anti mouse IgG (Sigma-Aldrich, M8642-1MG).

\section{Activity measurement on suspended PET}

Activity measurements of whole cell suspension $\left(\mathrm{OD}_{630}=0.1\right)$ surface-displaying PETase variants and free PETase enzyme (post TEV cleavage, corresponding roughly to a concentration of $0.01-0.1 \mu \mathrm{M}$ ) on a suspended, semi-crystalline PET powder (Goodfellow Co, ES306031) were executed by adapting a plate readerbased assay previously described [17]. In short, reactions were performed in sealed low binding 96-well plates (Greiner Bio-One $^{\mathrm{TM}}$ 655900), incubated at $40{ }^{\circ} \mathrm{C}$, in 50 $\mathrm{mM}$ sodium phosphate $\mathrm{pH}$ 8, at $1100 \mathrm{rpm}$ in an Eppendorf thermomixer for $4 \mathrm{~h}$. PET concentration was $15 \mathrm{~g} / \mathrm{L}$ and enzyme sample volume was $50 \mu \mathrm{L}$ in a total volume of $250 \mu \mathrm{L}$. Reactions were quenched by centrifugation, the supernatant withdrawn, and formation of soluble products was detected by measuring the absorbance at $240 \mathrm{~nm}$.

\section{Results and discussion}

Selection of membrane anchors for IsPETase surface display in E. coli

To overcome the bottleneck of inefficient heterologous expression and secretion of IsPETase variants in substantial amounts for screening and characterization, we adapted a surface display system previously described [14] to enable activity screening of IsPETase variants without the need for secretion into the growth medium. The system developed by Wendel et al. consists of both $\mathrm{N}$ - and C-terminal transmembrane anchors fused to the protein of interest. Sandwiched in between the membrane anchor and the protein of interest is a single domain camelid-derived antibody fragment (nanobody; NB) with high affinity for GFP [18], binding the fluorophore in a stable complex, thus allowing visualization of the surface display module when exposed on the cell surface. 
Two different membrane anchors were explored: (i) the C-terminal translocation unit of the Neisseria gonorrhoeae autotransporter IgA protease (C-IgAP) and (ii) a LppOmpA fusion protein consisting of the Lpp signal peptide followed by five transmembrane segments (residues 66 to 180) of $E$. coli outer membrane protein A (OmpA) (Fig. 1). All three versions of the IsPETase (Is PETase $^{\mathrm{WT}}$, IsPETase ${ }^{\mathrm{Austin}}$, and Is $\mathrm{PETase}^{\mathrm{Son}}$ ) were fused to the GFP-nanobody (part of the anchoring module) either C-terminally with a PelB signal peptide directly fused to the $\mathrm{N}$-terminus of the enzyme, or $\mathrm{N}$-terminally allowing a free $\mathrm{C}$-terminus, resulting in a total module size of 85 or $55 \mathrm{kDa}$, respectively. The two modules insert in antagonal orientation into the bacterial cell membrane. This way, Is PETase variants are oriented in opposite directions and can be translocated across the membrane without direct fusion to the signal peptide in the $\mathrm{N}$-terminal LppOmpA module. In both cases, protein production is under the control of the rhamnose-inducible rhaP $\mathrm{BAD}_{\mathrm{BAD}}$ promoter and successful display on the cell surface can be assessed upon GFP binding. This allows an easy and fast estimation of the relative surface display capacity. Furthermore, the introduction of TEV cleavage sites at both the $\mathrm{N}$ - and $\mathrm{C}$-termini (C-IgAP module) or solely the $\mathrm{N}$-terminus (LppOmpA module) along with a C-terminal histidine-tag prior to the TEV site allow the controlled release of the IsPETase variants into solution at any desired time point, as well as their subsequent purification.
The efficiency of the different transmembrane anchors for displaying the IsPETase was evaluated on whole cells. In short, this was done by isolating the cells post induction (expression at $30{ }^{\circ} \mathrm{C}$ ), and quantifying the signal derived from the GFP bound to the surface-exposed nanobody. This has previously been shown to be an excellent proxy for measuring total surface displayed protein [14]. The LppOmpA anchor successfully displayed the IsPETase-containing surface display modules, as confirmed via fluorescence measurement in a microtiter plate reader as well as via in-gel fluorescence of OD-normalized whole-cell samples after incubation with GFP (Fig. 2a). At non-denaturing conditions, concise bands corresponding to a complex of the surface display module bound to GFP via the nanobody element [theoretical sizes 112 (C-IgAP) and $82 \mathrm{kDa}$ (LppOmpA)] could be detected for the LppOmpA module, whereas no fluorescence appeared to originate from either the C-IgAP module or the unbound GFP $(27 \mathrm{kDa})$. The additional bands on the native gel most likely stem from partially denatured and incompletely or incorrectly folded LppOmpA modules containing the nanobody-GFP complex. Induced cultures were highly fluorescent compared to non-induced ones, with cultures expressing the LppOmpA anchor module emitting significantly higher fluorescence than the C-IgAP cultures. This suggests that the $\mathrm{N}$-terminal fusion construct is the most efficient for signal peptide-free IsPETase expression. Fusion of the pelB signal peptide directly onto the enzymes'

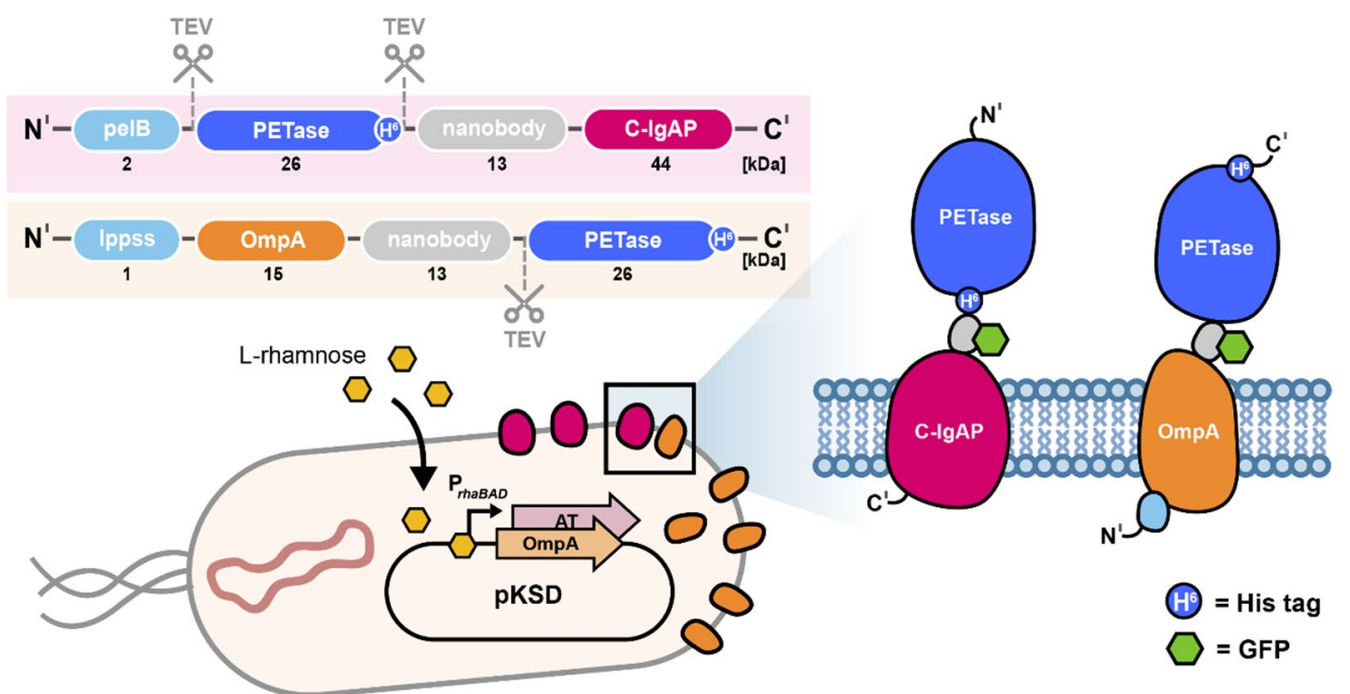

Fig. 1 Schematic illustration of surface display modules for ISPETase expression. C-IgAP (purple) and LppOmpA (orange) anchor module and orientation of IsPETases within the modules are shown. TEV cleavage sites and His-tags (6x histidine) are indicated. N-terminal signal sequences (Ippss and pelB) precede both constructs. A schematic illustrating both modules in the bacterial outer membrane is displayed (right side), indicating the location of nanobody:GFP (gray:green) binding. The pelB signal sequence is cleaved during translocation and therefore not displayed in the schematic 

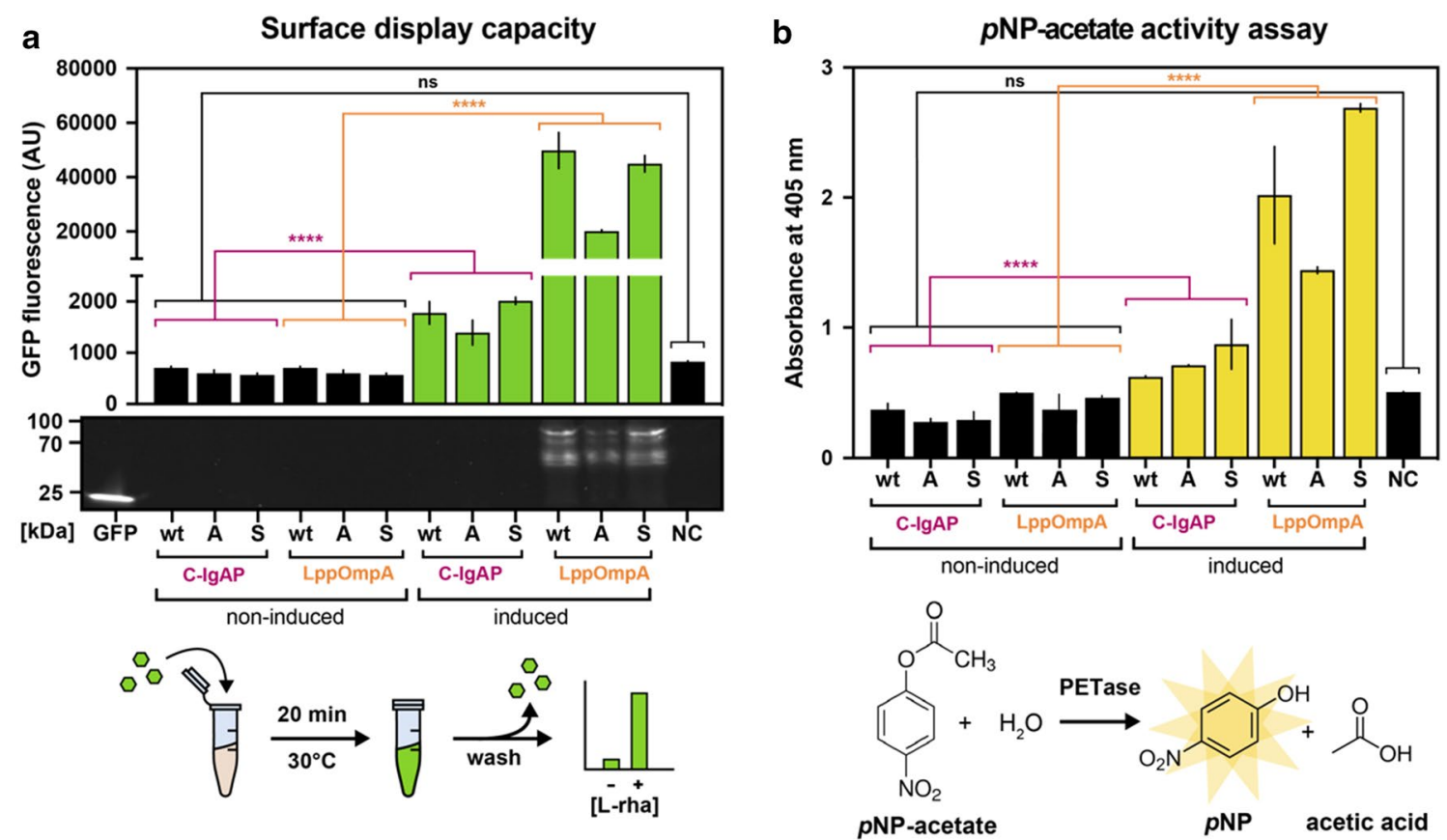

Fig. 2 Functional surface display of IsPETase variants comparing a C-IgAP and LppOmpA anchor module. a Whole-cell and in-gel fluorescence for IsPETase variants (wt: wild type; A: Austin, S: Son) surface-displayed using either C-IgAP or LppOmpA module with and without L-rhamnose induction. The nanobody:GFP binding workflow is indicated. Unbound GFP was run on the outer left lane. Samples have been normalized to ODU. b Absorbance change at $405 \mathrm{~nm}$ measured on pNP-acetate for IsPETase variants expressed via the two modules. Active PETase degrades $p N P$-acetate to $p N P$, and acetic acid as illustrated. NC: Negative control BL21(DE3) cells not harboring the expression vector. Statistical evaluation: Multiple comparison analysis (two-way ANOVA); ns: no significant statistical difference, ${ }^{* * * *}$ : p-value $<0.0001$

$\mathrm{N}$-terminus (C-IgAP module) was previously observed to be possible, but inefficient for IsPETase secretion [8] in line with our observations. A further advantage of the LppOmpA anchor module is the free C-terminus on the PETase, which readily allows fusion of tags or reporter proteins for downstream processing.

To investigate whether IsPETase enzymes displayed on the bacterial cell surface were correctly folded in an active conformation, we screened the six candidate constructs for activity on $p \mathrm{NP}$-acetate, a standard chromogenic model substrate for esterases [19]. For the surface display modules that were $\mathrm{N}$-terminally fused onto the LppOmpA fusion protein, and in good correlation with the GFP binding assay, higher amounts of released $p$ NP could be detected as a larger shift in absorbance (Fig. 2b). Identical results could be confirmed using the comparable compound $p$ NP-butyrate as substrate (Additional file 1: Figure S1). Despite the high background activity of the BL21(DE3) strain on the $p$ NP-acetate, a significant activity increase for all the membrane-bound IsPETase variants post induction could be detected when compared to non-induced cultures. Based on these observations, the LppOmpA anchor module was chosen for all further experiments.

\section{Surface-bound IsPETase ${ }^{\mathrm{WT}}$ is active on PET microparticles}

To eliminate the background activity detected for nonproducing $E$. coli cells on $p \mathrm{NP}$-acetate, as well as, to investigate whether the surface-bound IsPETase protein was folded correctly and in an active state, we next assessed the activity of the IsPETase ${ }^{\mathrm{WT}}$ towards PET particles. IsPETase ${ }^{\mathrm{WT}}$ exhibits several fold lower activity towards PET than improved enzyme variants. This makes the wildtype enzyme an ideal candidate to test both baseline sensitivity of the assay and suitability of a surface display strategy for detection of non-optimized PETases. To do so, cell-bound IsPETase ${ }^{\mathrm{WT}}$ produced in BL21(DE3) harboring expression vector pKSD:LppOmpA-NBIs PETase ${ }^{\mathrm{wt}}\left(20 \mathrm{~h}\right.$ post induction, expression at $\left.30^{\circ} \mathrm{C}\right)$ was prepared and surface display verified by activity towards $p$ NP-acetate (Fig. 3a) and GFP fluorescence (Fig. 3b).

Suspended, semi-crystalline PET powder, was used as substrate and the formation of degradation product was detected via an increase in absorbance at $240 \mathrm{~nm}$. As expected, no shift in absorbance (due to release of PET degradation products) could be detected for noninduced cells not expressing the IsPETase surface display module (Fig. 3c). However, significant IsPETase activity on free PET could be detected for the surface-displayed 

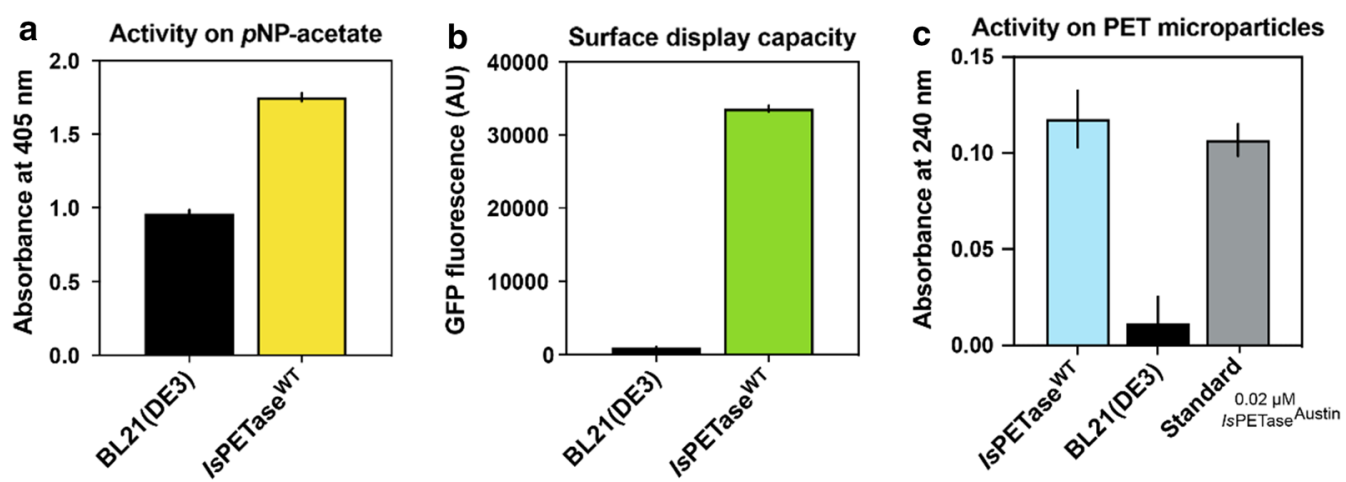

Fig. 3 Characterization of surface-bound IsPETase ${ }^{W T}$ activity. a Activity and $\mathbf{b}$ whole-cell fluorescence of BL21(DE3) cells expressing IsPETase ${ }^{\text {WT }}$ within the LppOmpA surface display module compared to BL21(DE3) grown without expression vector. c Degradation of PET microparticles $(15 \mathrm{~g} / \mathrm{L})$ measured via absorbance increase at $240 \mathrm{~nm}$ for wt $/ \mathrm{sPETase}$ bound to the bacterial cell surface. Standard: $0.02 \mu \mathrm{M}$ purified /sPETase Austin

IsPETase ${ }^{\mathrm{WT}}$ while still bound to the cell surface. The activity of the surface displayed PETase corresponded to the activity of $20 \mathrm{nM}$ purified (free) Is PETase ${ }^{\text {Austin }}$ measured under the same conditions [16] and used here as a positive control. This showed that the IsPETase ${ }^{\mathrm{WT}}$ was correctly folded and displayed in sufficient quantities for detection.

\section{Surface display allows expression and activity assessment of different IsPETase variants}

Having shown that the IsPETase ${ }^{\mathrm{WT}}$ was correctly folded when surface-displayed, we next wanted to explore the correlation between the surface-bound IsPETase activity measured in the initial screening on $p \mathrm{NP}$-acetate with the functionality of the free enzyme on its (actual) substrate PET. This was done by utilizing the TEV cleavage sites present in the anchor module to release all three surface-displayed IsPETase variants into the supernatant while removing the insoluble fraction by centrifugation. Measurements of the $p$ NP-acetate activity in the soluble fraction clearly showed that all three PETase variants had been successfully released from the cell surface via TEV treatment (Fig. 4a). The background activity previously observed for the BL21(DE3) control strain was now absent, indicating that it could be caused by a native $E$. coli membrane-associated esterase. Solubilization of the IsPETase variants by TEV cleavage resulted in an approximate 10-fold loss in absolute activity. This was most likely a combination of inefficient solubilization and, more significantly, removal of the high $E$. coli background activity which accounted for $86-87 \%$ of the total activity prior to TEV cleavage (Fig. 4a). TEV-mediated enzyme release thus enables detection of false positive (non-producing or inactive) batches, as it decreases the amount of background activity for the non-expressing $E$. coli production strain significantly. Additionally, the relative abundance of the three IsPETase variants either prior or post TEV cleavage was similar, confirming that activity on $p \mathrm{NP}$-acetate is not caused by unspecific activity of the $E$. coli background strain but derived from the surface-displayed IsPETase variants.

The three IsPETase variants chosen for surface display (IsPETase ${ }^{\mathrm{WT}}$, IsPETase ${ }^{\text {Austin }}$ and IsPETase ${ }^{\text {Son }}$ ) have previously been characterized $[7,9,11]$, and therefore, provide an optimal test set of enzymes to verify the screening strategy explored here. While the IsPETaseAustin variant (S238F and $\mathrm{W} 159 \mathrm{H}$ ) has improved PET degradation activity due to a narrowed binding cleft [9], Son et al. implemented three point mutations (S121E, $\mathrm{D} 186 \mathrm{H}$ and $\mathrm{R} 280 \mathrm{~A}$ ) into the wildtype enzyme increasing its thermal stability $\left(\mathrm{Tm}+8.81{ }^{\circ} \mathrm{C}\right)$, meanwhile, further enhancing the enzyme's PET degradation activity (14-fold at $40{ }^{\circ} \mathrm{C}$ ) [7] compared to Is PETase ${ }^{\mathrm{WT}}$. As the variants have been evolved towards higher specific activity on PET and not $p \mathrm{NP}$-acetate, it was expected that $p \mathrm{NP}$-acetate is unsuited to resolve clear activity differences (Fig. 4a). Nonetheless, initial activity screening on $p \mathrm{NP}$-acetate offers a fast and easy preliminary screening step to select active enzyme variants and reduce sample size for downstream analysis.

We next purified the solubilized IsPETase ${ }^{\mathrm{WT}}$ and Is PETase ${ }^{\text {Son }}$ using His-tag purification columns. The two PETase variants were chosen as they both appeared to be surface-displayed at similar levels as determined by GFP fluorescence (Fig. 4b). As a quality control, activity of the purified samples towards $p \mathrm{NP}$-acetate was measured. As shown in Fig. 4c, background activity was significantly removed during purification, supporting the earlier notion that this resulted from a native $E$. coli esterase. Even though enzyme amounts post purification were not in sufficient amount to be detected on 

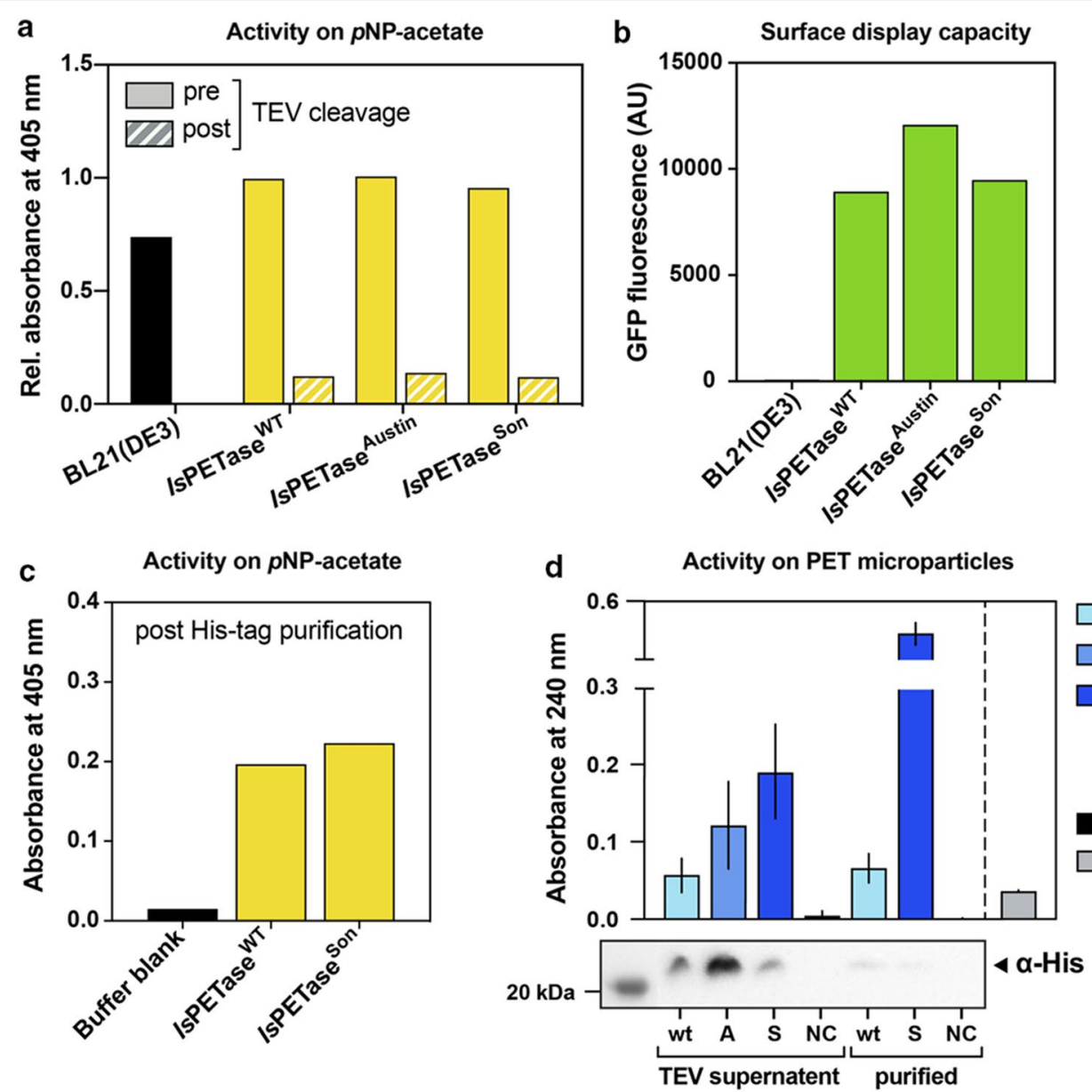

Fig. 4 Surface display and activity assessment of different ISPETase variants. a Activity of wt, Austin, and Son /sPETase variants expressed via the LppOmpA surface display module prior to and post TEV cleavage, hence release from the cell surface. To allow comparison of absorbance, values are relative to $/ s$ PETase ${ }^{W T}$ activity prior to TEV cleavage. Expression of all samples was performed at $16{ }^{\circ} \mathrm{C}$. $\mathbf{b}$ Whole-cell fluorescence verification of surface-displayed ISPETase variants (wt, Austin, Son) prior to TEV cleavage. c Activity of ISPETase ${ }^{W T}$ and IsPETase ${ }^{\text {Son }}$ on $p N P$-acetate after His-tag purification. Buffer blank: $50 \mathrm{mM}$ HEPES buffer. $\mathbf{d}$ Activity and detection of the previously described /sPETase variants expressed on the surface and subsequently cleaved off (TEV supernatant) and His-tag purified (purified). Activity of the released IsPETase variants on PET microparticles (upper half) and detection of the His-tagged enzymes via western blot (lower half) using an anti-His antibody. NC: Negative control BL21(DE3) cells

a SDS-PAGE, all IsPETase could be detected using Histag specific ( $\alpha$-His) antibodies via western blot (Fig. $4 \mathrm{~d})$. This was not surprising, as protein surface display suffers from the bottleneck caused by the inefficient and competition-heavy bacterial membrane translocation machinery, as well as the spatial limitation of the cell surface itself [20].

Interestingly, enzyme activity on PET increased after purification even though absolute enzyme amounts decreased, indicating inhibition by other proteins or compounds released during cell lysis present in the unpurified supernatant or better accessibility of the smaller enzyme to the corrugated surface upon removal of potential steric hindrance of the large construct
(Fig. 4d). This observation is supported by the presence of multiple proteins visible on the SDS-PAGE of Is PETase samples after TEV cleavage but prior to purification (Additional file 1: Figure S2). Activity on PET for the purified IsPETase ${ }^{\mathrm{WT}}$ enzyme was comparable to its activity in the TEV-cleaved but non-purified sample. In contrast, the purified IsPETase ${ }^{\text {Son }}$ exhibited a 2.5-fold increase in activity on PET compared to its non-purified sample, and a sevenfold higher activity compared to the purified wildtype enzyme. The latter corresponds well with the increased enzyme activity reported for IsPETase ${ }^{\text {Son }}$ [7] and supports the hypothesis of optimized substrate binding/enzyme efficiency post purification. Comparing band intensities visible 
on the western blot (Fig. 4d), the apparent protein concentration of the IsPETase ${ }^{\text {Son }}$ variant was approximately half of IsPETase ${ }^{\mathrm{WT}}$. This, combined with the observed 7 -fold activity increase on PET, adds up roughly to the 14-fold higher activity previously reported for the variant. Looking at the TEV supernatant of IsPETaseSon and IsPETase ${ }^{\text {Austin }}$, a similar comparison of protein concentrations in the western blot and activity towards PET (Fig. 4d) would suggest a lower specific activity of IsPETase ${ }^{\text {Austin }}$ at the conditions used.

A future refinement would be to include purified samples of selected enzymes, thereby allowing an improved quantification of the total amount of surface displayed enzyme. A potential caveat is that enzymes with large differences in specific activity (as seen for the IsPETase variants in this study) could easily bias this analysis. In contrast, using GFP binding as a proxy for quantifying the amount of surface display enzyme functions independently of enzyme specific activity. In summary, we show that an LppOmpA fusion protein can facilitate functional surface display of IsPETases and therefore easily be assayed whilst still bound to the cell surface, as well as after release into solution via the simplistic procedure outlined here. Two different surface display modules were investigated in this study, of which the spatial architecture of the LppOmpA module with the C-terminally positioned PETase resulted in the most optimal configuration. Further optimization of the culturing/expression conditions, the TEV cleavage, and the production host strain can be explored for more efficient expression of the IsPETase variants in this set-up. Surface display can be used for rapid screening of novel PETase variants enabling a fast and simplistic activity readout on PET. Moreover, the availability of the GFP nanobody allows assessment of expression independently from enzyme activity, which makes it well suited as initial screening tool for new enzyme candidates and allows for easy implementation into high-throughput screening workflows in both academic and industrial settings.

\section{Supplementary Information}

The online version contains supplementary material available at https://doi. org/10.1186/s12934-021-01582-7.

Additional file 1. Figure S1. Comparison of IsPETase activity on pNPacetate and -butyrate. Statistical evaluation: Multiple comparison analysis (two-way ANOVA), * $p$-value $<0.05,^{* * *}$ : $p$-value $<0.0001$. Figure S2. SDS-PAGE corresponding to western blot in Fig. 4c. S: Protein standard (Novex ${ }^{\text {TM }}$ Sharp, ThermoFisher), WT: IsPETase ${ }^{\text {WT }}$, A: IsPETase ${ }^{\text {Austin }}$, S: IsPETase ${ }^{\text {Son }}$, NCl: TEV buffer control, NC"l. BL21(DE3) wt control, NC III. HEPES buffer control. Table S1. Overview of primers. Primers used to construct vectors pKSD:LppOmpA-NB and pKSD:NB-C-IgAP via uracilexcision (USER) based cloning. Table S2. Sequences used in this study. All are based on the IsPETaseWT with the listed mutations in subscript.
Acknowledgements Not applicable.

\section{Authors' contributions}

$\mathrm{SAHH}$ performed the experiments that led to the creation of this article; analysed and interpreted the data for evaluation of the surface display modules and PETase activity on $p N P$-acetate. JAB performed the PETase activity assays on PET microparticles. SAHH and KJ were major contributors in writing the manuscript. MHHN and $\mathrm{KJ}$ contributed greatly to shaping the idea of the manuscript and the experimental design process. All authors revised the final manuscript. All authors read and approved the final manuscript.

\section{Funding}

This work was supported by the European Union's Horizon 2020 research and innovation program under grant agreement no. 887711 (Glaukos) to KJ, Novo Nordisk Foundation grant number NNFSA 170028392 to PW and Novo Nordisk Foundation grant number NNF170C0027752 to MHHN.

\section{Availability of data and materials}

Data sharing is not applicable to this article as no datasets were generated or analysed during the current study. The plasmid vector used for surface display is available from the corresponding author on reasonable request.

\section{Declarations}

Ethics approval and consent to participate

Not applicable.

\section{Consent for publication}

Not applicable.

\section{Competing interests}

The authors declare the following conflicts of interest: Kenneth Jensen works for Novozymes, a major manufacturer of industrial enzymes.

\section{Author details}

${ }^{1}$ Novozymes A/S, Biologiens Vej 2, 2800 Kgs. Lyngby, Denmark. ${ }^{2}$ Novo Nordisk Foundation Center for Biosustainability, Technical University of Denmark, Kemitorvet B220, 2800 Kgs. Lyngby, Denmark. ${ }^{3}$ Department of Biotechnology and Biomedicine, Technical University of Denmark, Søltofts Plads, 2800 Kgs. Lyngby, Denmark.

Received: 10 March 2021 Accepted: 20 April 2021

Published online: 01 May 2021

\section{References}

1. Carr CM, Clarke DJ, Dobson ADW. Microbial polyethylene terephthalate hydrolases: current and future perspectives. Front Microbiol. 2020;11:1-23.

2. Samak NA, Jia Y, Sharshar MM, Mu T, Yang M, Peh S, et al. Recent advances in biocatalysts engineering for polyethylene terephthalate plastic waste green recycling. Environ Int. 2020;145:106144.

3. Taniguchi I, Yoshida S, Hiraga K, Miyamoto K, Kimura Y, Oda K. Biodegradation of PET: current status and application aspects. ACS Catal. 2019:9:4089-105.

4. Hiraga K, Taniguchi I, Yoshida S, Kimura Y, Oda K. Biodegradation of waste PET. EMBO Rep. 2020;21:49826.

5. Danso D, Chow J, Streita WR. Plastics. Environmental and biotechnological perspectives on microbial degradation. Appl Environ Microbiol. 2019:85:1-14.

6. Yoshida S, Hiraga K, Takehana T, Taniguchi I, Yamaji H, Maeda Y, et al. A bacterium that degrades and assimilates poly(ethylene terephthalate). Science. 2016;351:1196-9.

7. Son HF, Cho IJ, Joo S, Seo H, Sagong HY, Choi SY, et al. Rational protein engineering of thermo-stable PETase from Ideonella sakaiensis for highly efficient PET degradation. ACS Catal. 2019;9:3519-26. 
8. Seo H, Kim S, Son HF, Sagong HY, Joo S, Kim KJ. Production of extracellular PETase from Ideonella sakaiensis using sec-dependent signal peptides in E. coli. Biochem Biophys Res Commun. 2019;508:250-5.

9. Austin HP, Allen MD, Donohoe BS, Rorrer NA, Kearns FL, Silveira RL, et al. Characterization and engineering of a plastic-degrading aromatic polyesterase. Proc Natl Acad Sci U S A. 2018;115:E4350-7.

10. Cui Y, Chen Y, Liu X, Dong S, Tian Y, Qiao Y, et al. Computational redesign of a PETase for plastic biodegradation under ambient condition by the GRAPE strategy. ACS Catal. 2021:11:1340-50.

11. Joo S, Cho IJ, Seo H, Son HF, Sagong HY, Shin TJ, et al. Structural insight into molecular mechanism of poly(ethylene terephthalate) degradation. Nat Commun. 2018;9:382

12. Liu B, He L, Wang L, Li T, Li C, Liu H, et al. Protein crystallography and sitedirect mutagenesis analysis of the poly(Ethylene terephthalate) hydrolase petase from Ideonella sakaiensis. ChemBioChem. 2018;19:1471-5.

13. Bloois E, Van, Winter RT, Kolmar H, Fraaije MW. Decorating microbes: surface display of proteins on Escherichia coli. Trends Biotechnol. 2011:29:79-86

14. Wendel S, Fischer EC, Martínez V, Seppälä S, Nørholm MHH. A nanobody: GFP bacterial platform that enables functional enzyme display and easy quantification of display capacity. Microb Cell Fact. 2016;15:1-13.

15. Nour-Eldin HH, Hansen BG, Nørholm MHH, Jensen JK, Halkier BA. Advancing uracil-excision based cloning towards an ideal technique for cloning PCR fragments. Nucleic Acids Res. 2006:34:e122-2.
16. Arnling Bååth J, Borch $K$, Jensen K, Brask J, Westh P. Comparative biochemistry of four polyester (PET) hydrolases. ChemBioChem. 2020 https://doi.org/10.1002/cbic.202000793.

17. Arnling Bååth J, Borch K, Westh P. A suspension-based assay and comparative detection methods for characterization of polyethylene terephthalate hydrolases. Anal Biochem. 2020;607:113873.

18. Kirchhofer A, Helma J, Schmidthals K, Frauer C, Cui S, Karcher A, et al. Modulation of protein properties in living cells using nanobodies. Nat Struct Mol Biol. 2010;17:133-8.

19. John GT, Heinzle E. Quantitative screening method for hydrolases in microplates using $\mathrm{pH}$ indicators: determination of kinetic parameters by dynamic pH monitoring. Biotechnol Bioeng. 2001;72:620-7.

20. Earhart CF. Use of an Lpp-OmpA fusion vehicle for bacterial surface display. Methods Enzymol. 2000;326:506-16.

\section{Publisher's note}

Springer Nature remains neutral with regard to jurisdictional claims in published maps and institutional affiliations.
Ready to submit your research? Choose BMC and benefit from:

- fast, convenient online submission

- thorough peer review by experienced researchers in your field

- rapid publication on acceptance

- support for research data, including large and complex data types

- gold Open Access which fosters wider collaboration and increased citations

- maximum visibility for your research: over $100 \mathrm{M}$ website views per year

At BMC, research is always in progress.

Learn more biomedcentral.com/submissions 